\title{
Some Applications of Chaos in Power Converters
}

\author{
David C. Hamill, Jonathan H.B. Deane and Philip J. Aston \\ School of Electronic Engineering, Information Technology and Mathematics \\ University of Surrey, Guildford GU2 5XH, United Kingdom
}

D.Hamill@surrey.ac.uk

\begin{abstract}
Applications are beginning to be found for chaotic power converters. Using the peak current controlled boost converter as an example throughout, the paper reviews the theory of chaos, shows how it may be employed to improve the electromagnetic compatibility (EMC) of power supplies, and presents a recently developed targeting scheme that can make a chaotic converter jump rapidly between two stabilised modes of operation.
\end{abstract}

\section{INTRODUCTION}

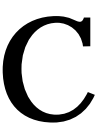

haos can be loosely defined as apparently random behaviour which is found in a wide variety of deterministic nonlinear systems. Since any circuit involving diodes is nonlinear, chaotic behaviour is expected to be widespread throughout power electronics. This has been borne out by numerous studies, which have revealed the possibility of chaotic operation in many power converters.

In an earlier colloquium [1] we described how chaos can occur in power converters. At that stage, work was confined to describing chaotic operation of dc-dc converters and explaining how the phenomenon could be modelled. Practical applications were only hinted at. In the intervening four years or so, considerable progress has been made in understanding the bifurcation phenomena of switched linear systems. Moreover, a whole new area of research, control of chaos, is being developed within the nonlinear dynamics community and has ramifications in power electronics.

This paper consists of three main parts, all based on the same example circuit, the boost dc-dc converter. First, we give a brief review of nonlinear dynamics ("chaos theory"). Next we investigate the use of chaos to improve the EMC of switched mode power supplies. Finally we present some recent results on the application of targeting theory to make a controlled chaotic converter jump between two stabilised modes of operation.

\section{REVIEW OF NONLINEAR DYNAMICS}

The erratic, bounded oscillations of chaos may seem like random noise, but they have a different origin. They are not caused by chance factors such as thermal noise, but are inherent in the equations underlying the ideal, noiseless system. In other words, chaos is deterministic: it is inherent in the solution of the system's differential equations, just as sine waves result from solving $d^{2} x / d t^{2}+\omega^{2} x=0$.

Though deterministic, the oscillations of chaos are unpredictable: their exact course cannot be known ahead of time. Chaotic systems are highly sensitive to perturbations, so the slightest error in the initial conditions will quickly blow up into a large error in the predicted waveform. This is what makes it impossible to predict the weather (the first chaotic system to be identified) more than a few days in advance. Despite their unpredictability, chaotic waveforms are bounded: they are confined to a particular region of state space called a strange attractor.

The peak current controlled boost dc-dc converter (Fig. 1) is a well known and widely used circuit for raising a dc supply to a higher voltage. Its waveforms are shown in Fig. 2. The switch closes when a clock pulse arrives. At this instant the inductor current is $i=i_{n}$ and the capacitor voltage is $v=v_{n}$. The inductor current starts increasing until it reaches the reference current $I_{\text {ref }}$ after time $t_{n}$. Note that zero, one or several clock pulses may occur during this interval. When $i$ reaches $I_{\text {ref }}$, the switch opens. It stays open until the next clock pulse, at which instant the current is $i_{n+1}$ and the capacitor voltage is $v_{n+1}$. (In a practical circuit there is usually an additional slower outer voltage loop, which sets $I_{\text {ref }}$ to regulate the output voltage.)

The circuit was analysed in [2], and a relationship linking $i_{n}$ and $v_{n}$ to $i_{n+1}$ and $v_{n+1}$ was found, of the form

$$
\left.\mid \begin{array}{l}
i_{n+1}=f_{1}\left(i_{n}, v_{n}\right) \\
v_{n+1}=f_{2}\left(i_{n}, v_{n}\right)
\end{array}\right\}
$$

or, in terms of the state vector $\mathbf{x}=\left[\begin{array}{ll}i & v\end{array}\right]^{T}$,

$$
\mathbf{x}_{n+1}=\mathbf{F}\left(\mathbf{x}_{n}\right)
$$

This is a nonlinear two-dimensional mapping, i.e. it transforms a point $\mathbf{x}_{n}$ in the two-dimensional state space to another point in the same space. It encapsulates all the vital properties of the circuit in a single equation. By iterating the mapping (applying it repeatedly, producing $\left.\mathbf{x}_{n+1}, \mathbf{x}_{n+2}, \mathbf{x}_{n+3}, \cdots\right)$, all the nonlinear behaviour of the real circuit can be emulated, including subharmonics and 


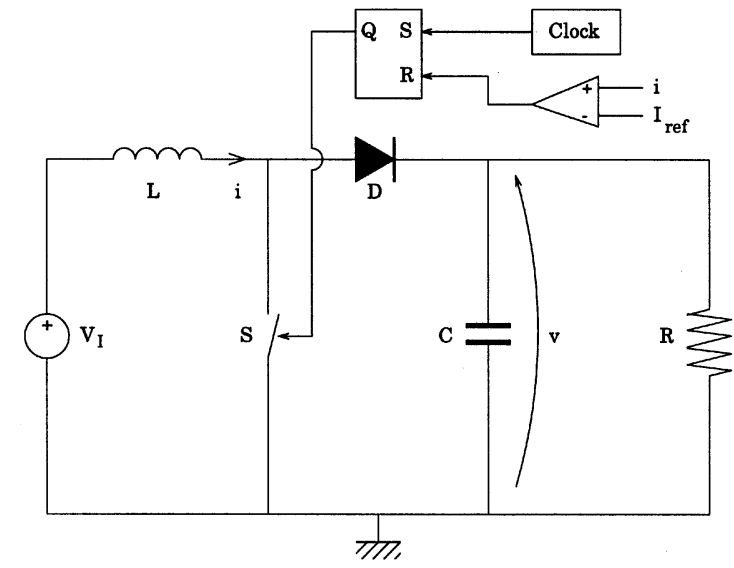

Fig. 1: Peak current controlled boost converter.

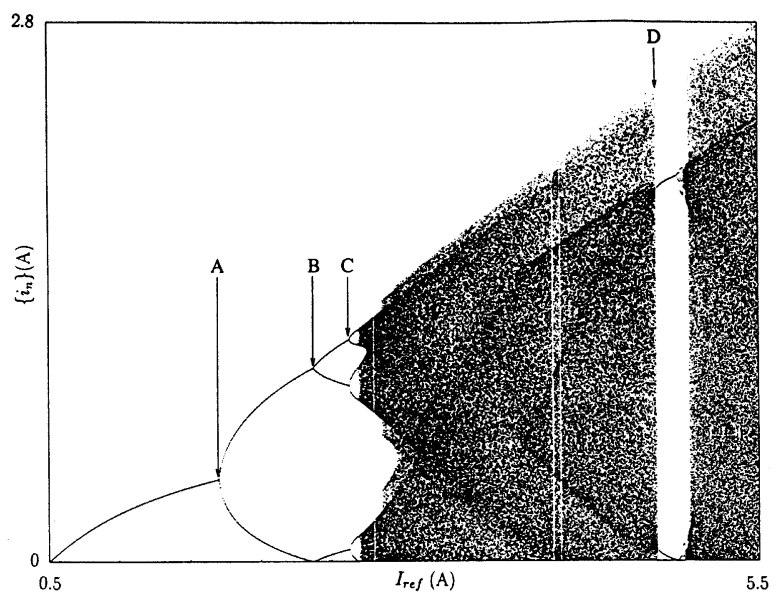

Fig. 3: Bifurcation diagram for the peak current controlled boost converter.

chaos [3]. If a special value $\mathbf{x}^{*}$ exists such that $\mathbf{F}\left(\mathbf{x}^{*}\right)=$ $\mathbf{x}^{*}$, it is called a fixed point of the mapping, because $\mathbf{x}_{n}=$ $\mathbf{x}_{n+1}=\mathbf{x}_{n+2}=\cdots=\mathbf{x}^{*}$.

The behaviour of the converter can be summarised compactly by means of a bifurcation diagram. For the boost converter, the following parameter values were used: $R=20 \Omega, L=1 \mathrm{mH}, C=12 \mu \mathrm{F}, T=10 \mu \mathrm{s}, V_{I}=10 \mathrm{~V}$, $I_{r e f}=0$ to 5.5A. In Fig. 3 the $i_{n}$ values, for $n=500 \ldots 750$, are plotted against the reference current $I_{r e f}$. When $I_{r e f}$ is just above zero, the fixed point is stable: after any initial transient the converter settles to a mode of operation where each cycle is same as the next (period-1). The dots for all the $i_{n}$ lie on top of each other, so as $I_{r e f}$ is increased a line is traced out. This is "normal, stable operation" or "the steady state", in conventional engineering terms.

However, as $I_{\text {ref }}$ is increased further, the fixed point becomes unstable at point $\mathrm{A}$ in Fig. 3. The behaviour bifurcates to a period-2 subharmonic, i.e., every other cycle is the same. This subharmonic oscillation is well known in current mode converters. If $I_{r e f}$ is increased further, bifurcations occur at points $\mathrm{B}$ and $\mathrm{C}$, to period-3 and period-6 subharmonics. Eventually chaotic operation is entered. Now each $i_{n}$ is different, producing a swarm of dots. At point D, a period-2 subharmonic appears.

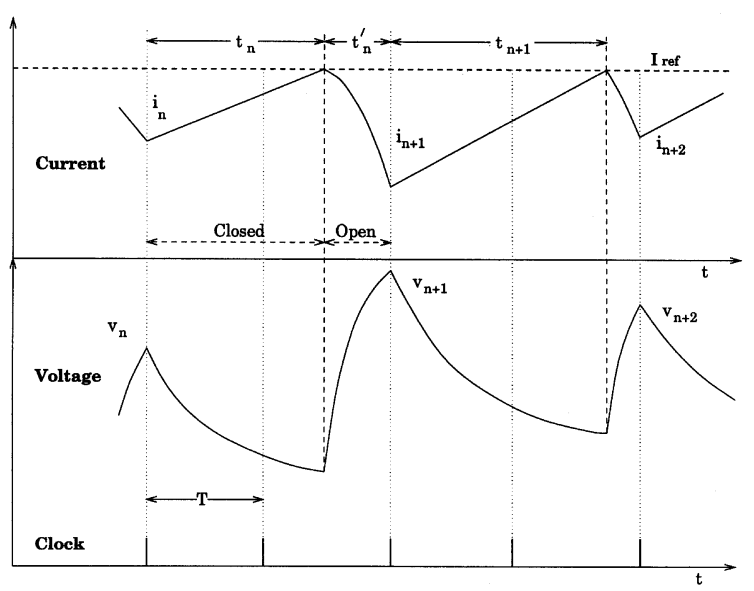

Fig. 2: Waveforms of the peak current controlled boost converter.

A recently discovered route to chaos, which may turn out to be prevalent in power converters, involves border collision bifurcations. Operation jumps suddenly from orderly to chaotic operation as a parameter changes slightly. This phenomenon is explained by the fixed point colliding with a borderline which corresponds to a discontinuity in the mapping [4].

\section{IMPROVEMENT OF EMC}

It is now a legal requirement that all equipment sold in the EU must comply with regulations on mains harmonic currents and radio frequency interference. In most cases this means that power supplies must be equipped with some form of high power-factor rectifier, in place of the capacitor input rectifier ubiquitous prior to these regulations. Most high power-factor rectifiers are based on the boost dc-dc converter (or similar), in which, as a side effect, a high ripple current at the switching frequency and its harmonics is injected into the mains via the input inductor. Thus in solving the low frequency mains harmonics problem, the high frequency interference problem has been worsened. One expert estimates that differential mode conducted interference can increase by $30 \mathrm{~dB}$ or more over a wide range of frequencies, while common mode interference is magnified by $6-10 \mathrm{~dB}$. It is especially difficult to ameliorate the common mode noise in two-wire (unearthed) equipment, as the only technique available is the common mode choke.

Since filtering has its limitations, other approaches have been tried. Because of the way the EMC regulations are drafted, wideband noise is more acceptable than narrow band. Some work has been done on spectral spreading by means of periodic or pseudo-random modulation of the clock frequency, and related methods. The purpose of spectral spreading is to reduced the measured interference peaks. This works because the same power is spread over a wider bandwidth, greater than the resolution bandwidth of the spectrum analyser specified in the standards. Although this may appear questionable, EMC compliance as enacted is what actually matters. A 


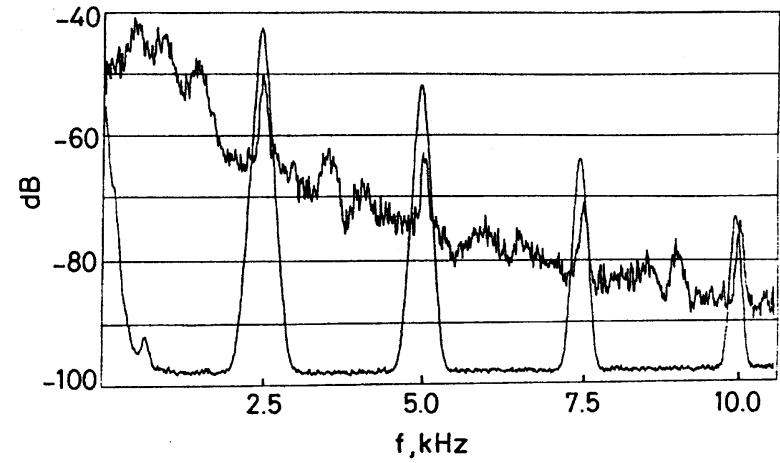

Fig. 4: Experimental spectra from the circuit of Fig. 1. The comb-like spectrum is for periodic behaviour, the broadband spectrum for chaos.

particular strength of spectral modification at source is that it affects all emission routes similarly: both radiated and conducted interference, the latter via both differential and common modes. In contrast, screening and filtering must utilise separate techniques for each route.

The techniques suggested for spectral spreading involve additional circuitry and therefore extra cost and complexity. On the other hand, chaos is a promising candidate. Having noiselike, aperiodic waveforms, it is characterised by a continuous, broadband spectrum though there may be spectral peaks. Chaos has the

TABLE I:

Reduction of Spectral Peaks by Chaos

\begin{tabular}{|c|c|}
\hline Harmonic number & Reduction (dB) \\
\hline 1 & 7.0 \\
\hline 2 & 11.0 \\
\hline 3 & 7.0 \\
\hline 4 & 0.0 \\
\hline 5 & 7.0 \\
\hline 6 & 6.0 \\
\hline 7 & -2.0 \\
\hline 8 & 5.0 \\
\hline 9 & 8.0 \\
\hline 10 & 4.0 \\
\hline 11 & -5.0 \\
\hline 12 & 4.0 \\
\hline 13 & 5.0 \\
\hline 14 & 0.0 \\
\hline 15 & 0.0 \\
\hline 16 & 5.0 \\
\hline 17 & 3.0 \\
\hline 18 & 0.0 \\
\hline 19 & 2.0 \\
\hline 20 & 4.0 \\
\hline Mean & 3.6 \\
\hline
\end{tabular}

advantage that it is inherent in boost converter circuits, so it can be introduced without a significant cost penalty. The spectral properties of chaotic power converters are not yet well understood, but initial results are encouraging and theoretical work is in progress to try and elucidate the processes involved.

To demonstrate the viability of the technique, the current controlled boost converter of Fig. 1 was investigated experimentally [5]. The parameter values were $R=$ $250 \Omega, L=100 \mathrm{mH}, C=220 \mu \mathrm{F}, T=400 \mu \mathrm{s}, V_{I}=10 \mathrm{~V}, I_{r e f}$ $=0$ to $0.5 \mathrm{~A}$. (The unrealistically low switching frequency of $2.5 \mathrm{kHz}$ was chosen to eliminate the effect of high frequency parasitics, making the converter closer to ideal and therefore more amenable to comparison with subsequent theoretical analysis.) With the feedback loop connected, the circuit behaved chaotically, i.e., the input current $i$ was aperiodic. The output voltage was about $29 \mathrm{~V}$ with a small ripple. The spectrum of $i$ was measured using an audio frequency spectrum analyser. The feedback loop was then disconnected and a pulse generator used instead to drive the switch periodically, with a duty ratio chosen so that the output voltage was again $29 \mathrm{~V}$. The current spectrum was re- measured. The two spectra are shown superimposed in Fig. 4.

The peaks in the spectrum occur at multiples of $1 / T$. Table I shows the amount by which chaos reduces them. Although some peaks are greatly reduced, by up to $11 \mathrm{~dB}$, others are emphasised by as much as $5 \mathrm{~dB}$. The average reduction of $3.6 \mathrm{~dB}$ represents a useful improvement, and this is without any attempt at optimisation. Reports of other spectral spreading methods suggest that 9-10dB may be possible. This ten-fold reduction in interference power would be difficult and expensive to achieve by filtering and screening. It may also be possible to tailor the chaos to diminish particular harmonics.

An alternative control circuit obtained by modifying the circuit of Fig. 1 guarantees chaos (at least in the idealised case). A D-type flip-flop is used instead of the set-reset type. Its CLK input is connected to the clock pulse generator, and its D input is fed from the comparator output. The switch is driven from the $\overline{\mathrm{Q}}$ output, with logic 1 meaning ON. Therefore, the inductor current $i$ is sampled at each clock instant. If $i \geq I_{\text {ref }}$ the switch is open until the next clock pulse; if $i<I_{r e f}$ it is closed until the next clock pulse. Thus the pulse sequence driving the switch consists of 0's and 1's, each an integer multiple of $T$ in duration. The inductor current is no longer peak controlled, but it is bounded nevertheless.

If the output capacitor $C$ is assumed infinite, the system is one-dimensional. The probability of a 1 is $p(1)=1$ $-V_{I} / V_{O}$, equal to the duty ratio for periodic operation. If $V_{o} / V_{I}$ is exactly rational, the sequence will repeat (possibly with a long cycle length). For instance, if $V_{O} / V_{I}=2$ the sequence will be $1,0,1,0, \cdots$, or if $V_{o} / V_{I}=3$, the sequence will be $1,1,0,1,1,0, \cdots$. However, the set of rational numbers is infinitesimal compared to the real numbers, and noise is always present, so in practice repetitive sequences will be intermittent. If there is 


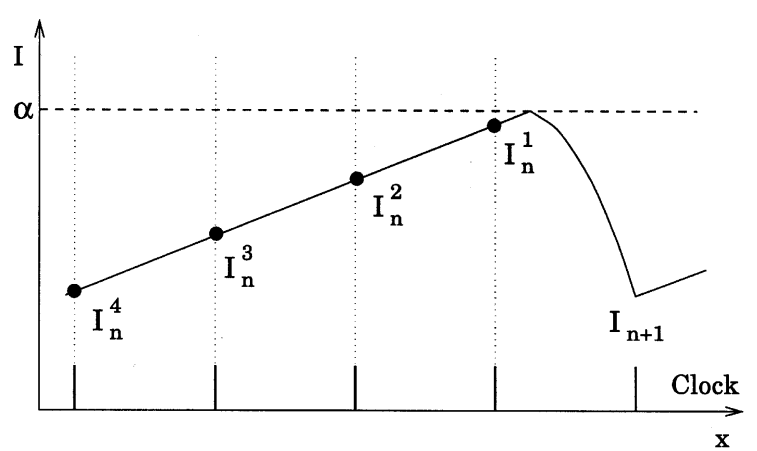

Fig. 5: Because $i$ can be less than $I_{\text {ref }}$ for several clock pulses, the value $I_{n+1}$ has multiple inverses, $I_{n}{ }^{4}$ to $I_{n}{ }^{1}$.

inductor resistance or if $C$ is finite, making the system two-dimensional, bifurcations to periodic operation may occur.

A problem facing the designer of a chaotic spectral improvement scheme is therefore to ensure that chaos occurs under all operating conditions. Another problem is that the total noise power with chaos might be greater than without. Third, undesirable low frequency components are present. It is hoped that statistical theory may assist in suggesting some remedies. However, the application of statistics to chaotic waveforms is still in its infancy.

\section{STABILISATION AND CONTROL}

Control methods for chaotic systems are being developed, based on the fact that a small perturbation can have large repercussions (the "butterfly effect"). Pioneering work by Ott, Grebogi and Yorke [6] stabilised a saddle fixed point contained in a chaotic attractor by applying small parameter variations. A saddle fixed point possesses a stable manifold (a line of maximum contraction) as well as an unstable manifold (a line of maximum expansion). Equation (2) may be modified to

$$
\mathbf{x}_{n+1}=\mathbf{F}\left(\mathbf{x}_{n}, p\right)
$$

where $p$ is some controllable system parameter, e.g. $I_{\text {ref }}$ in the boost converter. The stabilisation technique works by waiting until the chaotic trajectory passes close by the fixed point $\mathbf{x}^{*}$, then changing $p$ so the next iterate lies on (or close to) the stable manifold. Subsequently only small perturbations in $p$ are needed to keep the trajectory from wandering away from the stable manifold. The method is based on a linear approximation to the dynamics near the fixed point, so it is only valid for small perturbations $|\delta p| \leq \delta p_{\max }$. One problem is that there can be a long chaotic transient before control is established; another is that control can be lost in the presence of noise (such as switching noise). Control schemes more suited to power converters have been suggested, e.g. [7].

Targeting schemes take this idea one stage further. They let a chaotic system jump rapidly from one stabilised fixed point $\mathbf{x}_{1}^{*}$ to another, $\mathbf{x}_{2}^{*}$ (the target), by applying a suitable $\delta p$. For instance, in the boost converter the

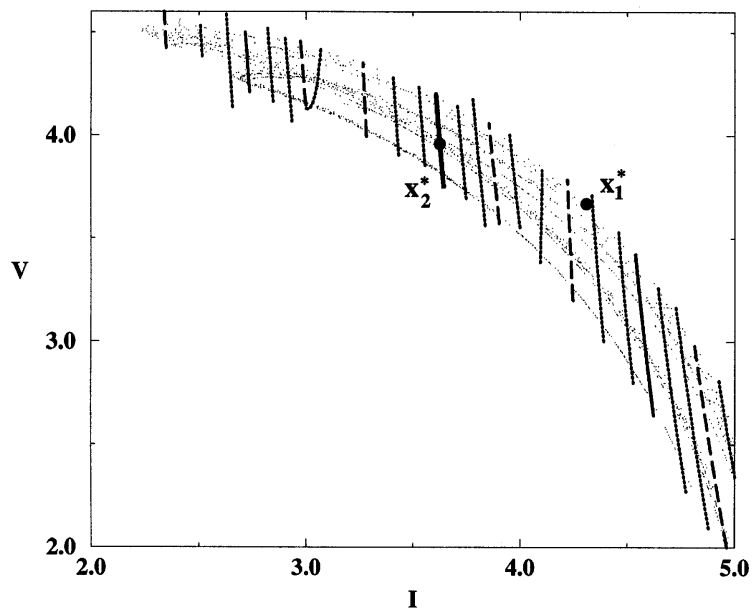

Fig. 6: A segment of the stable manifold through the target fixed point $\mathbf{x}_{2}{ }^{*}$ (heavy line) and its first, second and third pre-iterates. One of the 18 third pre-iterates lies close to the present fixed point, $\mathbf{x}_{1}{ }^{*}$.

current state might be a period- 2 subharmonic, and the desired state might be period-1, or vice versa. The original methods of targeting [8] rely upon forward iteration of a line segment consisting of $\mathbf{F}\left(\mathbf{x}_{1}^{*}, p\right)$ for all permissible values of $p$ and back-iterating a region around the target point $\mathbf{x}_{2}^{*}$ until an overlap is found. An appropriate parameter perturbation $\delta p$ can then be determined which, when applied at the first iteration, will direct the trajectory to the target region in a few iterations. At this point a control procedure can again be applied.

Switched-mode circuits give rise to discontinuous mappings which have multiple inverses, i.e. $\mathbf{F}^{-1}\left(\mathbf{x}_{n}\right)$ is multi-valued. This can be seen from the inductor current waveform of Fig. 6, where $I_{n}^{1}, I_{n}^{2}, I_{n}^{3}$ and $I_{n}^{4}$ are all inverses of $I_{n+1}$. Each of these values may itself have multiple inverses. Taking the target to be the stable manifold of the fixed point to be stabilised, only a few back iterations are required until pre-iterates of this manifold are spread over the attractor. When a pre-iterate lies close to the present fixed point, a parameter perturbation can be determined which will put the first iterate onto the preiterate of the stable manifold. The target fixed point will then be reached in a few iterations. Note that this method does not require forward iteration of a line segment.

This is illustrated for the peak current controlled boost converter in Fig. 6. The heavy line lies along the stable manifold of the target $\mathbf{x}_{2}^{*}$ (a period-2 subharmonic). One of its 18 possible third pre-iterates is seen to lie very close to $\mathbf{x}_{1}^{*}$ (a period-1 fixed point). Let $f$ be a line segment through $\mathbf{x}_{1}^{*}$ comprising the points accessible from $\mathbf{x}_{1}^{*}$ in a single iteration for the range of permissible values of $p$. The proper value of $\delta p$ can be determined from the intersection of $f$ with a pre-iterate of the stable manifold of $\mathbf{x}_{2}^{*}$ (see Fig. 7). Fig. 8 shows the trajectory followed when a suitable $\delta p$ is applied. After only five iterations, the target is approached closely, at which point a stabilisation scheme could be applied. A similar procedure can be performed to jump back to the original 


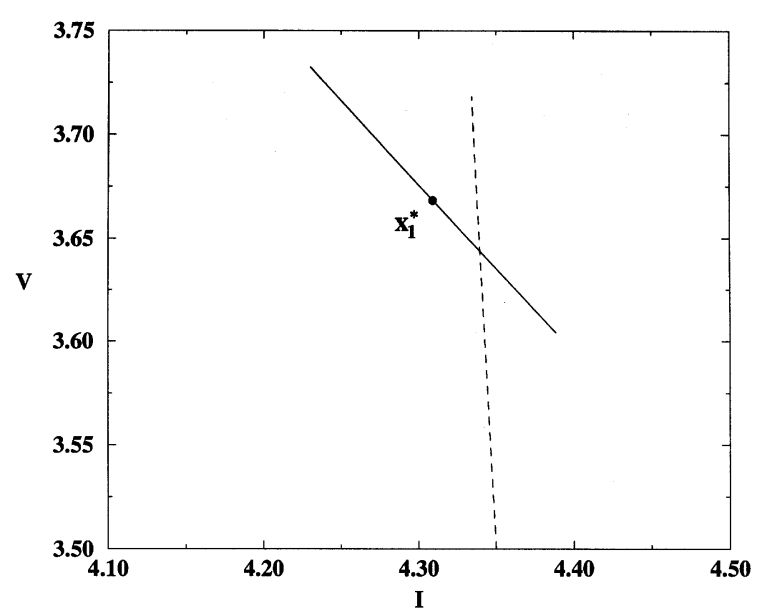

Fig. 7: Intersection of the line $f$ (solid line) and a third pre-iterate of the stable manifold of the target $\mathbf{x}_{2}{ }^{*}$ (broken line).

\section{fixed point.}

The benefits of this method are that it is not necessary to forward-iterate a controllable region and that the backiterates quickly cover the attractor, due to the multiple inverses. It seems that power converters may be inherently well suited to targeting methods. However, this work is in its early stages, and there are several practical issues to be confronted, such as the effect of switching noise.

\section{CONCLUSION}

Chaos in power electronics has been an area of intensive study for the last ten years or so. Although many interesting results have been discovered, there is still much that is at best poorly understood.

Following earlier work which focused on the modelling and bifurcation performance of power converters, research is now beginning to move towards uses for chaos. The major applications seem at present to be

- the possibility of improvement of EMC by chaotic spread spectrum techniques;

- targeting and control of chaos in switched mode power supplies;

- the increased agility of converters that are operating on the edge of stability.

All three are at an early stage of development, so the

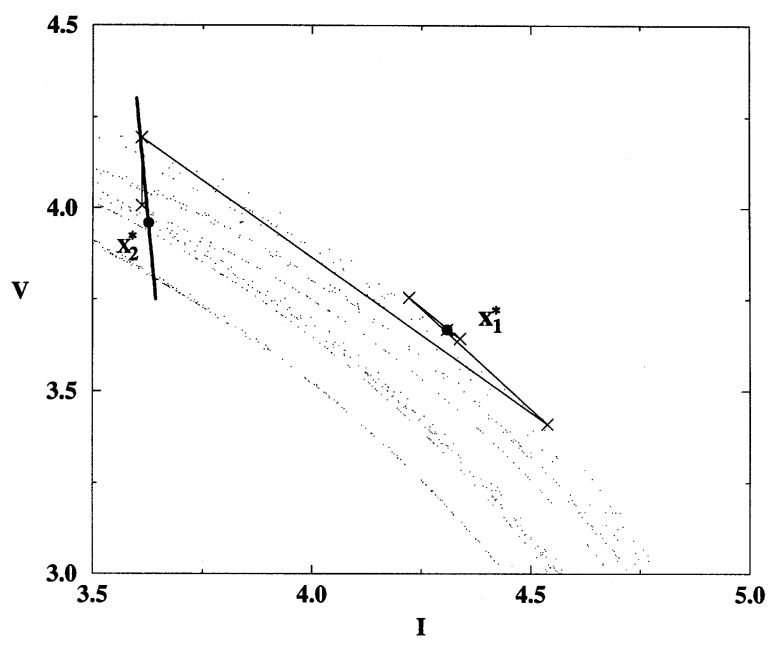

Fig. 8: The trajectory taken from $\mathbf{x}_{1}{ }^{*}$ to $\mathbf{x}_{2} *$ when the targeting scheme is applied. The target is approached closely after five steps.

future of chaos research in power electronics is likely to be exciting.

\section{REFERENCES}

[1] D.C. Hamill, "Chaos in dc-dc converters", IEE colloquium on static power conversion, IEE digest no. 1992/203, pp. 5.1-5.4, London, Nov. 1992

[2] J.H.B. Deane, "Chaos in a current-mode controlled boost dc-dc converter", IEEE Trans. on Circuits and Systems, Part I, vol. 39, no. 8, pp. 680-683, Aug. 1992

[3] D.C. Hamill, J.H.B. Deane and D.J. Jefferies, "Modeling of chaotic dc-dc converters by iterated nonlinear mappings", IEEE Trans. on Power Electronics, vol. 7, no. 1, pp. 25-36, Jan. 1992

[4] S. Banerjee, C. Grebogi and E. Ott, "Border collision bifurcation: an explanation of the observed bifurcation phenomena in the boost converter" (preprint, 1997)

[5] J.H.B. Deane and D.C. Hamill, "Improvement of power supply EMC by chaos", Electronics Letters, vol. 32, no. 12, p. 1045 , June 1996

[6] E. Ott, C. Grebogi and J.A. Yorke, "Controlling chaos", Phys. Rev. Letters, vol. 64, pp.1196-1199, 1990

[7] G. Poddar, K. Chakrabarty and S. Banerjee, "Control of chaos in the boost converter", Electronics Letters, vol. 31, pp. 841-842, 1995

[8] T. Shinbrot, E. Ott, C. Grebogi and J.A. Yorke, "Using chaos to direct trajectories to targets", Phys. Rev. Letters, vol. 65, pp. 3215-3218, 1990

[9] P.J. Aston, J.H.B. Deane and D.C. Hamill, "Targeting in systems with discontinuities, with applications to power electronics" (preprint, 1997) 